\title{
Mucus models to evaluate nanomedicines for diffusion
}

\section{Anne-Claire Groo ${ }^{1,2}$ and Frederic Lagarce ${ }^{1,3}$}

${ }^{1}$ LUNAM Université, INSERM U1066 MINT (Micro et nanomédecines biomimétiques), Angers, France

${ }^{2}$ Ethypharm, Grand-Quevilly, France

${ }^{3}$ Pharmacy Department, Angers University Hospital, Angers, France

In the fast-growing field of nanomedicine, mucus is often the first barrier encountered by drug products in the body, and can be the only barrier if it is not overcome by the drug delivery system. Thus, there is a need to design new nanomedicines that are able to diffuse easily across mucus to reach their pharmacological targets. In this design process, mucus diffusion studies are mandatory and have an important role in the selection of the best drug candidates. However, there is currently no standard procedure for diffusion studies across mucus. In this Foundation Review, we discuss the differences observed within mucus models and experimental protocols in diffusion studies, with an emphasis on nanomedicine diffusion.

\section{Introduction}

Colloids are being increasingly developed and used to enhance the efficacy, and reduce the toxicity, of drugs. In this promising area of so-called 'nanomedicine', some new drug formulations have already reached the market [1] and there is a substantial amount of research underway into new colloidal formulations to enhance their use as drug delivery systems. For example, encapsulation in nanodevices such as liposomes or nanocapsules can help the drug to have the desired distribution in the body, thus enabling it to reach its pharmacological target in sufficient concentration and avoiding other tissues where it can be toxic. Encapsulation also helps the drug to overcome biological barriers, such as the intestinal epithelium or the blood-brain barrier. The journey of a colloidal carrier in the body is complex and has been reviewed recently [2]; however, it often starts with an encounter with mucus. In fact, mucus is a complex biological material that lubricates and protects many tissues. Given that mucus is ubiquitous, colloid systems are in contact with it in many areas of the body, including lungs, gastrointestinal tract, vagina, eyes and nasal tract. Thus, it is necessary to characterize nanoparticle behavior in mucus during the process of formulation design and optimization.

Irrespective of its origin, mucus comprises water (approximately 95\%), glycoproteins (i.e. mucins), lipids $(0.5-5 \%)$, mineral salts $(0.51 \%)$ and free proteins (1\%) [3]; however, mucus displays different properties and fine composition depending on its location in the body. It inhibits penetration by numerous viruses [4] and is a useful barrier against other pathogens. However, mucus also constitutes a potentially efficient barrier to the delivery of nano-sized drug

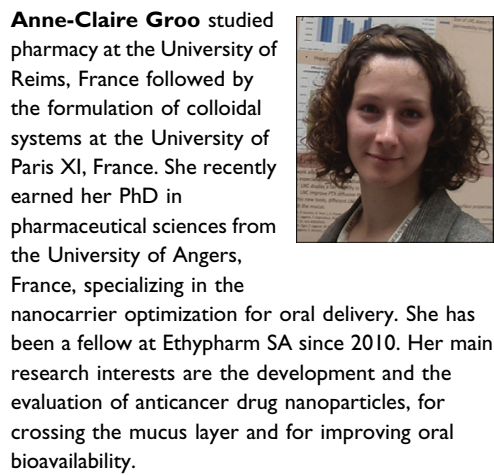

Anne-Claire Groo studied pharmacy at the University of Reims, France followed by the formulation of colloidal systems at the University of Paris XI, France. She recently earned her $\mathrm{PhD}$ in pharmaceutical sciences from the University of Angers, France, specializing in the nanocarrier optimization for oral delivery. She has been a fellow at Ethypharm SA since 2010. Her main research interests are the development and the evaluation of anticancer drug nanoparticles, for crossing the mucus layer and for improving oral bioavailability.

Frederic Lagarce received his $\mathrm{PhD}$ in 2004 and, since 2012 , has been a professor of pharmaceutical technology and biopharmaceutics in the University of Angers, France. $\mathrm{He}$ is also a hospital pharmacist, and so his research has a translational focus (from bench to bedside). His main

interest is in cancer therapy, especially bioavailability enhancement by exploiting the interactions between drug products (mainly nanosystems) and living tissues. This field involves not only biological barrier-crossing studies, but also

stability assessments of active moieties. Finding new answers to medical needs using innovative drug formulations is what drives him to work every day. 
delivery systems. Thus, it is of primary importance to design nanocarriers that are able to cross mucus, therefore, mucus diffusion studies are needed. Despite this need, there is currently no standard protocol available for mucus diffusion studies; here, we determine whether the different models used to study nanocarrier diffusion in mucus are similar enough to each other to provide approximately the same results or if there is a need for standardization. We first evaluate differences and similitudes of mucus models described in the literature. We then go on to compare diffusion models and evaluate the impact of experimental conditions on diffusion. Finally, we highlight how physicochemical properties of nanoparticles influence their diffusion through mucus.

\section{Mucus models}

The different models

There are various models of mucus described in the literature (Table 1), from the simplest ex vivo model to the closest in vivo models, and from simple mucin, artificial mucus, to natural mucus from horse, pig or human. It is also possible to use pathologic mucus and mucus produced by specialized cells for in vitro transcellular crossing or uptake experiments. Ex vivo or in vivo models have also been described.

The simplest models include only mucin solutions reconstituted with different solutes. Norris and Sinko prepared reconstituted mucin by mixing mucin with sodium phosphate and a sodium carbonate buffer, adjusted to $\mathrm{pH} 6.5$ [7]. By contrast, Dawson et al. prepared reconstituted artificial pig gastric mucus by mixing pig gastric mucin (PGM) $60 \mathrm{mg} / \mathrm{mL}$, dipalmitoylphosphatidylcholine (DPPC), bovine serum albumin (BSA) and Hepes buffer ( $\mathrm{pH} 7.4$ ) [12]. Bhat et al. prepared a model of cystic fibrosis mucus (CFM) by adding calf thymus DNA and BSA to reconstituted pig gastric mucus solution [16], whereas Bhat prepared a reconstituted pig gastric mucus solution by mixing PGM $(40 \mathrm{mg} / \mathrm{mL})$ and isotonic phosphate buffer ( $\mathrm{pH}$ 7.4) containing sodium azide, followed by two rounds of centrifugation and then dialysis [58]. Larhed et al. prepared an artificial mucus model comprising purified PGM $(0.4 \%)$, a lipid mixture (3\%), pig serum albumin (3.1\%), DNA $(0.5 \%)$, Tween $80(0.75 \%)$ and $10 \mathrm{mM}$ phosphate buffer [13].

Thus, these different preparation protocols resulted in different mucus models of differing $\mathrm{pH}$ and with different physicochemical properties. In fact, mucus models prepared from diluted mucin using different methodologies differed not only from each other, but also from crude mucus models extracted from animals.

\section{Advantages and limits of the models}

Crude mucus is the most ideal model but it has some disadvantages. First, it is difficult to access a source of mucus from an individual. Second, the composition and, thus, chemical and

TABLE 1

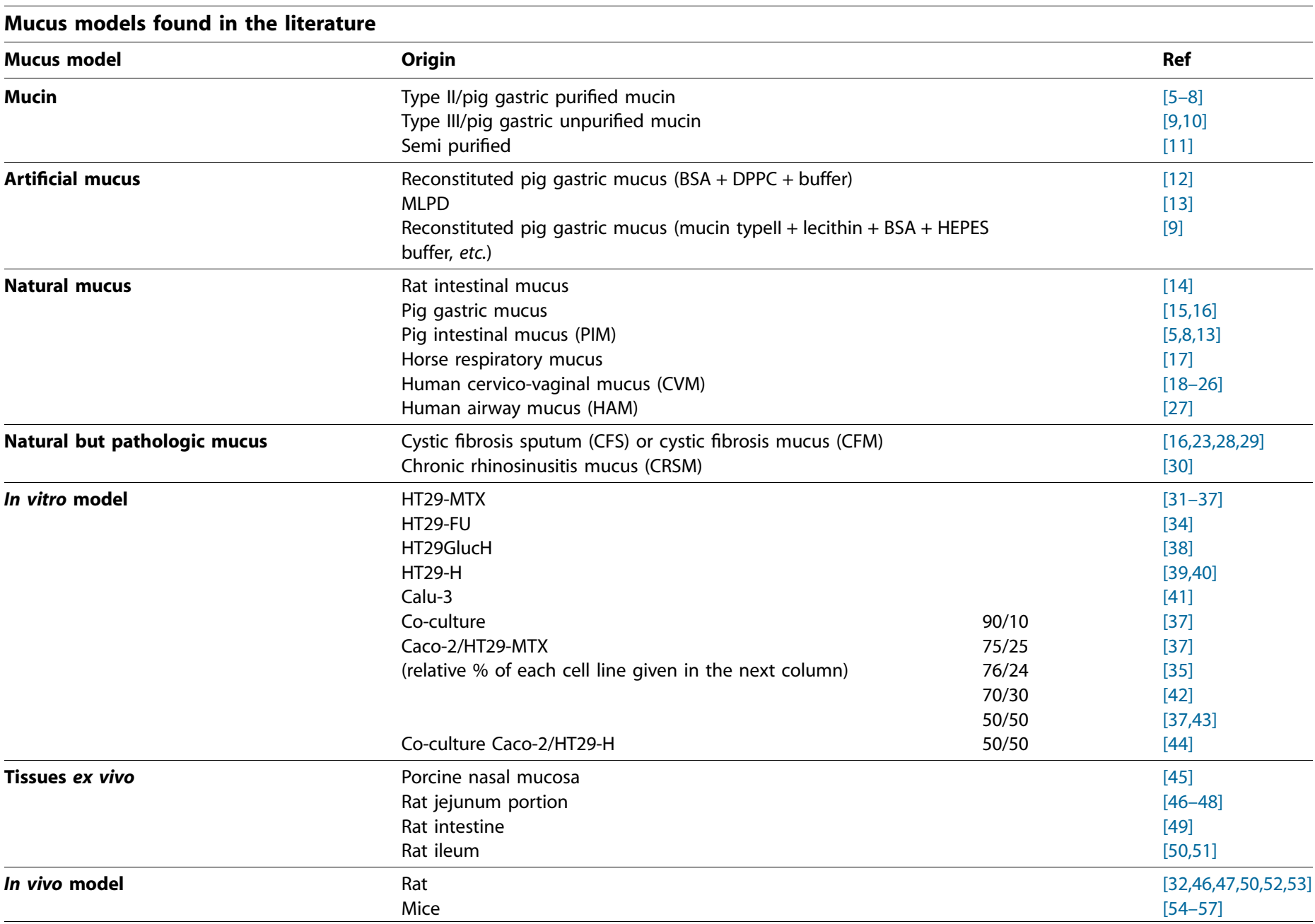


physical properties can vary between batches because of interindividual variability; for example, mucin concentrations varied from $10 \pm 2$ to $47 \pm 3 \mathrm{mg} / \mathrm{mL}$ among six different mucus samples [29]. To limit interindividual variation, some batches can be collected and mixed. Mucus samples can be stored at $-20^{\circ} \mathrm{C}$ without significant change. For example, no effect of freezing and thawing on viscoelasticity [29] was observed and storage at $-20^{\circ} \mathrm{C}$ did not influence the diffusion coefficients of drugs [5]. However, a little evaporative loss from cervicovaginal mucus $(\mathrm{CVM})$ was observed at room temperature and storage at $-20^{\circ} \mathrm{C}$ reduced evaporative loss [59].

Pig is a relatively large animal and so it is possible to obtain sufficient mucus from only few animals to perform several experiments. Moreover, pig mucus and human mucus are similar in structure and molecular weight [60], which is important given that it is also possible to observe differences in mucus properties for different animals of the same species [61]. As a result, commercial pig mucin has been used as a mucus substitute to prepare mucin solutions. Two forms of PGM are currently available commercially: purified mucin (type II) and unpurified mucin (type III). The main advantage is that the composition is more stable, although this is not always relevant given that mucus contains various other components, such as lipids, proteins, or salts. Thus, some researchers have focused on reconstituted mucus; for example, Dawson et al. [12] mixed PGM with BSA, DPPC and buffer, whereas McGill and Smyth [9] mixed mucin with lecithin, BSA and HEPES buffer; an artificial mucus model was also proposed by Larhed [13]. Interestingly, reconstitution of mucus with mucin did not suppress its variability compared with crude mucus, in that McGill and Smyth observed some heterogeneity in composition and nonuniformity of the rehydrated mucin polymers used in in vitro prepared mucus models [9].

Griffiths et al. demonstrated that the extraction process modifies commercial PGM samples. It disrupts the disulfide bridges, leading to a weaker sol-gel transition at around $\mathrm{pH} 4$ and a lack of gel formation [10]. The structural perturbation in mucin was confirmed by the lack of interactions between mucin and poly(ethylene glycol) (PEG). Bhat et al. compared drug diffusion through CFM and through gel and sol fractions generated by a separation process. Unfortunately, this process altered the mucin structure, as evidenced by the highly branched structures observed by transmission electron microscopy (TEM) [16].

In vivo and ex vivo assays can be consuming in terms of the number of animals required. However, working with slaughterhouses enables large amounts of animal mucosa to be obtained from only a few animals. For example, Wadell et al. studied diffusion through porcine nasal mucosa [45]. This mucosa was large enough and only one individual was used to achieve tissue specimens for a full six-chamber experiment, whereas a higher number of smaller animals were needed to get the same area. However, for studies involving intestinal mucosa, fewer animals are needed.

\section{Model comparisons}

Studies compared molecule diffusion through different mucus models and showed different apparent permeability according to the mucus model. Mucus composition depends on the origin of the mucus (species and organ source) and its composition influences its properties and reactivity against others molecules.
The weight-average molecular mass of mucin comprising mucus is different depending on the function of its original location [62]. For example, the molecular mass of pig colonic mucin is $5.5 \times 10^{6} \mathrm{Da}$, of human cervical mucin is $11 \times 10^{6} \mathrm{Da}$ and of PGM is $44 \times 10^{6} \mathrm{Da}$. The influence of mucus model composition on mucus properties has largely been studied over the past few years, with obvious differences reported between pathogenic and nonpathogenic models. For example, chronic rhinosinusitis mucus (CRSM) and cystic fibrosis sputum (CFS) have similar barrier properties because the viscoelasticity of mucus gel is exacerbated in both cases by pathogenic inflections and chronic inflammation. As a consequence, results of Lai et al. suggested that CRSM has greater adhesivity compared with healthy CVM [30]. The solution environment also has an impact on mucus properties, particularly in the case of disease. For example, in CFM, the high extracellular $\mathrm{Ca}^{2+}$ concentration leads to thick mucus over the long term [63]. The poor bicarbonate availability in this mucus can explain its high viscosity and mucin aggregation, because of the ability of bicarbonate to sequester $\mathrm{Ca}^{2+}$ [64]. The high levels of soluble proteins on the mucosa partially explain the characteristically thick mucus in asthma and other bronchial inflammatory diseases [65].

Purified PGM solution did not provide an accurate model of native mucus because it did not exactly reflect mucus constituents such as water, mucin and lipids, mineral salts and free proteins in either their quality or quantity, which increased the possible interactions between particles and mucus. Larhed et al. studied the diffusion of different drugs through native pig intestinal mucus (PIM) and purified pig gastric mucin (PPGM) [5]. They demonstrated that the diffusion coefficient of lipophilic drugs was reduced in a native PIM model but less so in PPGM, compared with the diffusion coefficient in buffer. For example, 36\% of cyclosporine A diffused in PPGM and 16\% in PIM. The same phenomenon was observed with another drug: 78\% of 1-deamino-8-D-arginine-vasopressin (dDAVP) diffused in PPGM and only $17 \%$ in PIM. Thus, a substantial part of the mucus barrier was likely to be formed by other components of the native mucus besides mucin. Moreover, a relation between lipophilicity (i.e. $\log P$ ) and diffusions in PIM was observed but no relation was found in PPGM. Thus, the native PIM was a more realistic model of gastrointestinal mucus and provided more information regarding the barrier properties of mucus in vivo. Similarly, McGill and Smyth's study showed that rhodamine B permeation was significantly different in mucin solution and in an artificial CFS model because of the differences in the composition of the mucus model [9].

Components other than mucin have also been found to be responsible for the reduced diffusion of lipophilic drugs in PIM compared with PPGM. Larhed et al. identified lipids and proteins as components with an important impact on drug diffusion [13], with the drugs interacting with the lipids and proteins. The same authors tried to reproduce artificial mucus with a composition mimicking PIM. However, the diffusion obtained in their artificial mucus was similar but not identical to that in PIM, highlighting the fact that it is difficult to reconstitute the complete structure of native mucus.

Bhat et al. compared the diffusion of three drugs through buffer, native mucus and synthetic mucus models [16]. For the drugs 
tested, the observed permeability was always in the following rank order: buffer $>$ reconstituted pig gastric mucus (i.e. mucin solution) $>$ whole $\mathrm{CFM}>\mathrm{CFM}$ sol fraction $>$ synthetic $\mathrm{CFM}>\mathrm{CFM}$ gel fraction. Synthetic CFM solutions were prepared by adding BSA and DNA to pig gastric mucus solutions. Lieleg et al. observed that mucin concentration was an essential parameter for diffusion: if mucin concentration increased, the impact of the barrier became more pronounced [66].

Mucin concentration can vary with the location of mucus in the body, as well as with various other physiological and pathological parameters [67]. Griffiths et al. studied the diffusion of polymers in mucin solutions that ranged in concentration from 0 to $5 \%(\mathrm{w} / \mathrm{w})$. A decrease in the diffusion rate was shown with increasing mucin concentration [10]. Therefore, diffusion depends largely on mucus components and its proportions, which can be explained by the fact that interfiber spacing depends only weakly on hydration but more on the concentration of mucin [21].

In addition to observed differences between mucus model compositions, differences in mucus model structure have also been observed and have a role in particle and drug diffusion. The average pore size of human CVM, determined by fitting the measured diffusion rates of particles to Amsden's obstructionscaling model, was $340 \pm 70 \mathrm{~nm}$ [18]. The average mesh spacing of human CFS was $140 \pm 50 \mathrm{~nm}$, as shown by the dynamics of mucus-resistant particles [28]. However, comparison is difficult because results depend on the source of mucus as well as on the method of sample preparation, which can disrupt mucus structure. Ensign et al. observed variations in the mucus mesh at different anatomical locations [68].

The sampling method used is also important, given that mucus comprises two layers, the firmly adherent mucus and the loosely adherent mucus [69]. If the extraction method is too energetic, the sample will contain remnants of mucosa, such as cells and DNA, whereas, if the sample is too superficial, it might contain only the free mucus layer.

The increased hydration of ovulatory endocervical mucus (OCM) compared with other mucus secretions (during nonovulatory periods and at other mucosal tissues) increased the pore size of mucin. Tang et al. performed studies of PLGA nanoparticle diffusion in OCM and CVM [23]. Differences between OCM and CVM led to a difference in the penetration improvement resulting from the PEG coating. A modest increase in penetration rate was observed in OCM, whereas the same modification improved the penetration rate in CVM 400-fold.

As discussed above, mucin is often used to prepare mucin solutions or artificial mucus, although the preparation methods used to obtain mucin can disrupt its structure. Therefore, even if mucin is used at the same concentration as in natural mucus and with the other components of mucus, differences can be observed compared with crude mucus. For instance, anionic particle mobility was significantly higher in purified PGM than in native intestinal mucus [8]. The difference in mesh structure of native mucus compared with that of purified mucin and/or differences in composition between the two media were related to this difference in mobility. The degradation occurring during the purification procedure has been related to one or other of these differences. Mucin was present at high concentrations in both media, and cationic particle mobility was similar, owing to the adhesion of the cationic particles to negatively charged mucin fibers. Particle transport rates were more heterogeneous in native mucus, because of the higher heterogeneity of the porosity of the mucus mesh. The more homogeneous nature of the purified mucin solution versus native mucus was supported by microscopic observation.

Nanoparticle transport behavior was not significantly different in colonic mucus on the surface of freshly excised mouse colon tissues compared with mucus scraped from the tissue surface. This suggests that the collected mucus layer was, in this case, not disturbed [68], and enabled the researchers to study the mucus barrier effect only.

\section{Diffusion systems}

\section{The different models}

Various protocols have been developed to evaluate interactions between particles and mucus (Table 2), such as the mucoadhesion assay $[48,70,71]$, in vivo experimentations $[32,55]$ with radioactivity studies [53] or pharmacokinetic (PK) studies [46,54,72], binding proprieties [73] and diffusion studies. Here, we focus on diffusion protocols and briefly discuss mucoadhesion, which is often the first step of diffusion.

To study drug and particle diffusion, numerous systems have been used, including multiple particle tracking (MPT) $[8,12,18$ $20,22,23,25,28,74-76]$; two samples tubes that are then filtered and centrifuged [11]; side-by-side systems [6,16]; side-on-three compartment diffusion [15]; diffusion chambers [45] including Ussing chambers $[46,47,49,77]$; modified Franz diffusion cells [14]; modified Transwell-Snapwell ${ }^{\mathbb{R}}$ diffusion chambers $[7,29]$; modified diffusion cell setups [9]; fluorescence recovery after photobleaching (FRAP) [21]; radioactivity with two syringes $[5,13]$; Transwell ${ }^{\circledR}$ covered by cells $[24,41,78]$; Transwell ${ }^{\circledR}$ diffusion [32,33,35-39,42,43,50]; and cell association [79].

Side-on-three compartment diffusion is one of the most commonly used methods. The diffusion cell comprises one donor compartment, one acceptor compartment and one central compartment containing the mucus model. Drugs or particles are placed in the donor compartment and their arrival in the acceptor compartment is evaluated over time. Given that mucus is placed between two compartments, the amount of drug or particles in this compartment determines their permeability or diffusion coefficient through the mucus.

Membranes between compartments are impermeable to mucus but not to drugs or particles; thus, their capacity to retain the drug or particles must be well known to distinguish the effects of the membrane versus the mucus diffusion. Therefore, different sideon-three compartments have been developed, in the form of diffusion chambers (i.e. side-by-side ${ }^{\mathbb{R}}$ diffusion cells) customized with a membrane holder (Fig. 1) $[6,16]$.

Numerous research teams have developed in-house manufactured side-on-three compartment diffusion cells. For example, Shaw et al. added a polycarbonate filter membrane and metal gauze filters to a diffusion cell [15]. Norris and Sinko modified Transwell-Snapwell ${ }^{\mathbb{R}}$ chambers, comprising two compartments and a tissue between them, by adding filters and a ring on which to place mucus [7], as did Sanders et al. [29]. Similarly, Grubel and Cave modified a microfiltration device to obtain a permeability device [11]. When studies focus on mucosal tissue, the experimental systems used are simpler because tissue is more easily 
maintained between compartments. For example, Bravo-Osuna et al. used a Ussing cell comprising two compartments separated by rat intestinal epithelium [77], whereas Wadell et al. placed porcine nasal mucosa in diffusion chambers [45].

Another approach was used by Lai et al. [19]. Particle transport rates were measured by analyzing trajectories of fluorescent particles in mucus bulk, by MPT. In this context, only one compartment is needed, thus avoiding the membrane affecting diffusion. The microscopic motion of hundreds of fluorescent particles is recorded by video microscopy and, thus, particle detection is performed in mucus without disturbing the system. In the same way, FRAP [21] has been used to investigate the mobility of labeled molecules in mucus and biogels. The sample is placed on a microscope and a high-intensity laser beam is used to bleach the fluorescence of the molecules, which causes a drop in the fluorescence intensity. The diffusion coefficient is obtained by the recovery profile of the fluorescence intensity, which results following diffusion of the nonbleached molecules into this area (Fig. 2) [80].

As a noninvasive method, pulsed-gradient spin-echo NMR (PGSE-NMR) was used by Griffiths et al. [10] to quantify the diffusion of a probe polymer. The central feature of this technique is the application of a magnetic field gradient that encodes the position of the molecule into the NMR signal.

Other original models can be used to predict particle diffusion through mucus. For example, a 2D model was developed for studying the interaction of surface-modified lipid nanocapsules (LNC) with mucus. This 2D model, based on surface balance measurements at a constant pressure or area, can be used as a screening method for choosing suitable surface-modified LNC formulations for assessing diffusion using 3D models [81].

\section{Advantages and limits of diffusion systems}

The limits of diffusion protocols are related to the quantification or detection methods used. Some teams have used radiolabeled drugs and detected resulting radioactivity by using a liquid scintillation counter. For example, Bravo-Osuna et al. used ${ }^{14} \mathrm{C}$ mannitol [77] and Wadell et al. used ${ }^{14} \mathrm{C}$ mannitol and $\mathrm{D}-(2-3 \mathrm{H})$ glucose [45], whereas Larhed et al. used ${ }^{14} \mathrm{C}$ mannitol, ${ }^{3} \mathrm{H}$ propranolol, ${ }^{14} \mathrm{C}$ hydrocortisone, ${ }^{3} \mathrm{H}$ testosterone and other radiolabeled drugs $[5,13]$. By contrast, radiolabeled ${ }^{14} \mathrm{C}$ ibuprofen was used by Shaw et al. [15]. In addition, Saltzman et al. labeled molecules with fluorescein and measured their diffusion coefficients by using computer imaging of fluorescence profiles and by FRAP [21]. However, working with radioactivity is expensive and is not easily accessed because of the need for specific equipment; in addition, agreement and safety rules are both necessary and stringent.

Much equipment is required for MPT, including a silicon-intensified target camera mounted on an inverted epifluorescence microscope equipped with a $100 \times$ oil-immersion objective lens; the appropriate filters; glass chambers; specific software; and

TABLE 2

Evaluation of colloid diffusion with different models related to their in vivo efficacy (predictability)

\begin{tabular}{llllll}
\hline Route & In vitro model & Ex vivo model & In vivo model & Ref & Drug nanocarrier \\
\hline Oral & Caco-2/HT29-M6 & Efficiency & {$[82]$} & $\begin{array}{l}\text { Encapsulation of calcitonin } \\
\text { into chitosan nanocapsules }\end{array}$
\end{tabular}

Predictability/conclusions

In vitro model revealed that the mucoadhesive properties of chitosan nanoparticles may represent a key factor for their ability to improve peptide absorption after oral administration

Ussing chambers In vivo [47] Paclitaxel (PTX)-loaded (rat jejunum) bioadhesive study and pharmacokinetic (PK)

Mucin adhesion (mucin type III) PK study

Muco-adhesion PK study pegylated nanoparticles (NP) Similar improvement of bioavailability was observed for PEG PTX-NP in vitro and in vivo

[83] PTX-loaded chitosan-vitamin E succinate (CV) nanomicelles (chitosan thiolated or not) Enoxaparin loaded nanocomplexes (chitosan grafted glyceryl monostearate copolymers)
Thiolation improve fourfold AUC for CV nanomicelles and lead only to a twofold increase in mucin adhesion Mucoadhesion results are not showed (only: mucoadhesion significantly increased with modification of chitosan with GM compared with that of chitosan, GM graft ratio: $3.7 \%=11.1 \%>18.6 \%$ and chitosan $100 \mathrm{kDa}>20 \mathrm{kDa})$

Vivo: $\mathrm{C}<3.7 \%<11 \%>18 \%$ and $100 \mathrm{kDa}>50 \mathrm{kDa}$ Same conclusion: maximal bioavailability for nanocomplexes prepared using $\mathrm{CS} 100$ GM11.4\% copolymers

\begin{tabular}{ll}
\hline Colon $\quad$ MPT in mucus & $\begin{array}{l}\text { Tracking on freshly } \\
\text { excised mucosal } \\
\text { tissues }\end{array}$
\end{tabular}

Pulmonary MPT in CFS
[68]

In vivo airway
gene transfer

$[85]$

[85] DNA nanoparticles composed of plasmid DNA compacted with block copolymer of polyL-lysine and PEG (2, 5, and $10 \mathrm{kDa})$
Displacement of particle were not significantly different in collected colonic mucus and in ex vivo colon tissue

All DNA nanoparticles were immobilized in freshly CFS.

Mice receiving $\mathrm{CK}_{30} \mathrm{PEG}_{10 \mathrm{k}}$ or $\mathrm{CK}_{30} \mathrm{PEG}_{5 \mathrm{k}}$ DNA nanoparticles exhibited higher luciferase expression than $\mathrm{CK}_{30} \mathrm{PEG}_{2 \mathrm{k}}$ (due to higher nuclease attack of $\mathrm{CK}_{30} \mathrm{PEG}_{2 \mathrm{k}}$ ) 
fluorescent particles. Lai et al. [18,19] and Suk et al. [28] also used fluorescent polystyrene (PS) particles obtained from molecular probes. Using the same method, Crater and Carrier used fluorescent FluoSpheres ${ }^{\mathbb{R}}$ obtained from Invitrogen molecular probes [8]. Tang et al. [23] prepared fluorescent PLGA nanoparticles. Unfortunately, fluorescent labeling can modify carrier properties and, thus, their diffusion ability.

In addition to quantification or detection methods, the diffusion system has a role in diffusion evaluation. Experimental variability was observed by Bhat et al. [16] using a modified side-by-side diffusion cell and mucus placed in an approximately 3 -mm thick chamber. This thickness is higher than is found in vivo. With a similar system, Norris and Sinko observed the same variability in the measurements with a smaller mucus thickness of $0.38 \mathrm{~mm}$ [7]. The variability was not only related to the thickness of mucus, but also to the complexity of the diffusion system. In fact, given that the membrane is a supplementary barrier to diffusion, its choice is important. The goal should be to select a membrane with the lowest effect on diffusion to observe phenomena purely related to mucus. In their study, Bhat et al. showed that drug diffusion was under membrane control for the three drugs that they tested [6]. In the case of diffusion across compartments, when the donor solution is placed in the donor compartment at the start of the experiment, the empty membrane becomes soaked by solution, corresponding to the time of flow establishment [86]. Steady-state conditions are then established. Membrane thickness should be the lowest and experimentation duration should be high enough to overlook the time of flow establishment. Experimentation duration should also be long enough to enable membrane equilibration, but also short enough to avoid significant concentration variations in the donor solution. In such a steadystate condition, equation of permeability can be simplified. Using these particular conditions, Bhat et al. determined permeation at steady state for drug diffusion across CFM [16].

The apparent permeability $P_{\text {app}}$, expressed in $\mathrm{cm} \mathrm{s}^{-1}$, is classically calculated using Eq. (I) $[7,14,45,51,77]$ :

$P_{\text {app }}=\frac{\mathrm{dQ}}{A C_{0} \mathrm{~d} t}$

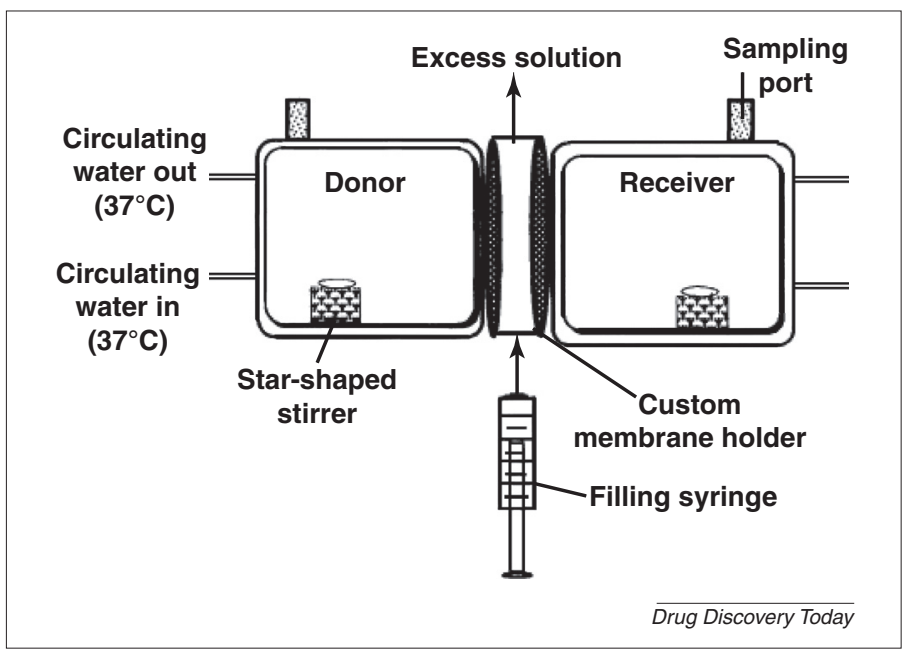

FIGURE 1

Side-by-side diffusion cell with a customized membrane holder.

Figure reproduced, with permission, from Bhat et al. [6].

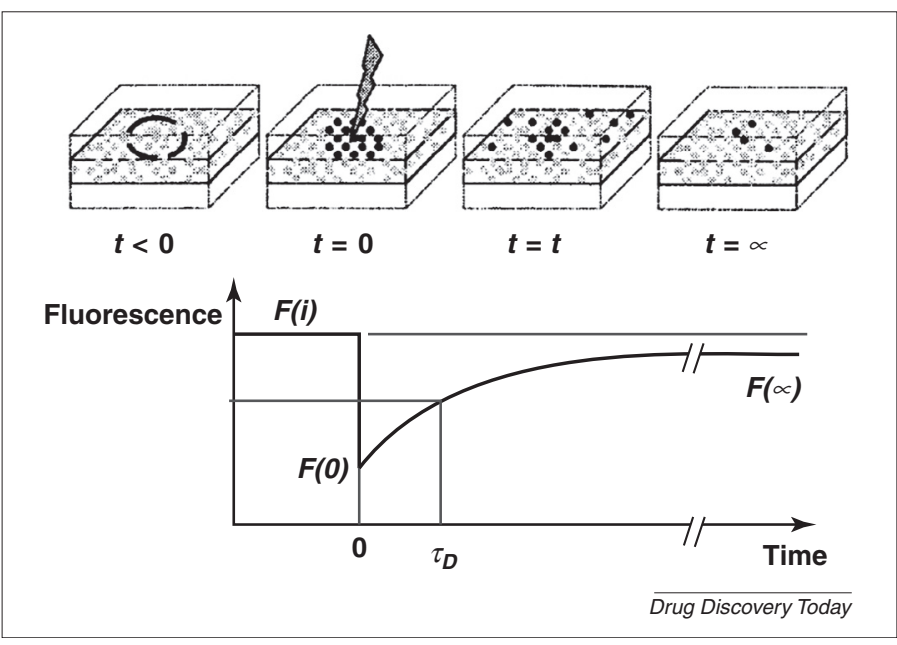

FIGURE 2

Schematic representation of a fluorescence recovery after photobleaching experiment. The initial fluorescence before bleaching is recorded as $F(i)$. At $t=0$, a drop in fluorescence to $F(0)$ is caused by a high-intensity light beam bleaching the molecules. The bleached molecules exchange their position in the bleached area with non-bleached fluorescent molecules from the surrounding, due to the random motion/diffusion. This results in a recovery of the observed fluorescence.

Figure reproduced, with permission, from Occhipinti and Griffiths [80].

where $\mathrm{dQ} / \mathrm{d} t$ is the rate of drug appearance on the received side $\left(\mu \mathrm{g} \mathrm{s}^{-1}\right), C_{0}$ is the initial concentration over the donor side $\left(\mu \mathrm{g} \mathrm{mL} L^{-1}\right)$ and $A$ is the surface area $\left(\mathrm{cm}^{2}\right)$.

It is important to note that Eq. (I) is valid and can be used only if the membrane volume is negligible versus the volume of the compartments; that is, $\lambda<0.02$. However, if either the length of membrane equilibration or membrane volume is too high, Eq. (I) should be modified using $\lambda$ [87].

Some researchers have used Eq. (II), which is based on this previous formula, but that takes into account the contribution of each barrier $[6,16]$. In case of multilayer systems, membrane permeability, $P$, is given by Eq. (II):

$P=\frac{D}{\delta}$

$\frac{1}{P_{\text {total }}}=\sum \frac{1}{P}=\sum \frac{\delta_{i}}{D_{i}}$

where $D$ is the diffusion coefficient of the molecule, expressed in $\mathrm{cm}^{2} \mathrm{~s}^{-1}$. The thickness of the layer $\delta$ is expressed in cm. A side-onthree compartment system contains two membranes and a mucus layer, the former of which can constitute an important barrier to diffusion [88]. In this case the permeability can be expressed as:

$\frac{1}{P_{\text {total }}}=\frac{1}{P_{\mathrm{mb} 1}}+\frac{1}{P_{\mathrm{mb} 2}}+\frac{1}{P_{\text {mucus }}}$,

where $P_{\mathrm{mb} 1}$ and $P_{\mathrm{mb} 2}$ represent the permeability across each of the membranes, $P_{\text {mucus }}$ is the permeability across the mucus layer and $P_{\text {total }}$ is the permeability across the entire system.

In the case of a lack of system uniformity, a concentration gradient appears and constitutes a pseudo-membrane [89]. The existence of unstirred layers implies that, in any phenomenon depending on the difference between the two surface concentrations, possible serious errors can be made by using the difference between the bulk concentrations. The effect of the unstirred layer 
is more complicated than the case of stirred layer, and is given by Eq. (IV):

$\frac{1}{P_{\text {total }}}=\sum \frac{1}{P_{\text {mb }}}+\frac{1}{P_{\text {mucus }}}+\sum \frac{1}{P_{\text {conc }}}$,

where $P_{\text {conc }}$ is the permeability across the pseudo-membrane together with the concentration gradient. For example, Korjamo et al. observed that, by varying stirring speed, the concentration gradient had a different effect on drug permeability across Caco-2 cells on Transwell ${ }^{\mathbb{R}}[90]$.

The method becomes very inaccurate when diffusion is almost entirely rate controlled by the unstirred layers [91]. This is why, if the concentration in each compartment varies substantially between the start and end of the experiment, stirring is necessary to homogenize the media.

\section{Parameters influencing diffusion and comparisons}

Diffusion in mucus depends on its composition [13], such as the mucin concentration [66], which, as discussed above, depends on the mucus model used [8]. Thus, the choice of mucus model is crucial. The mucus model used needs to be the closest to the type of physiological mucus encountered by the drug delivery system in vivo. Particle diffusion also depends on the surface chemistry of the particle [8] and the particle size [22]. However, other parameters can also influence diffusion and should be checked.

Grubel and Cave observed that the effect of the formulation on mucus viscosity appeared to determine the movement of clarithromycin through mucus [11]. For example, the greater proportion of inactive polymeric ingredients in Biaxin ${ }^{\circledR}$ granules, the higher the increase in the viscosity and the more enhanced the barrier properties of gastric mucin. Moreover, Sanders et al. showed that the elastic modulus of mucus influenced the percentage of transported nanospheres [29].

When Griffiths et al. added dendrimers to mucin solutions, changes in the mucin scattering were induced, indicating an interaction between these polymers and mucin [10], a change that was $\mathrm{pH}$ dependent. As a consequence, the diffusion of polymers showed a complex dependency on both $\mathrm{pH}$ and mucin concentration. Cao et al. demonstrated that mucus undergoes a pH-induced conformation change [92], whereas Lieleg et al. showed that increasing the mucus $\mathrm{pH}$ from 3 to 7 resulted in a general increase in particle mobility because acidic mucus formed a higher and more selective barrier compared with neutral mucus [66]. Given that mucus $\mathrm{pH}$ varies with its function and, therefore, localization in the body (Fig. 3), the choice of mucus source to set up a model has to be made carefully.

Shaw et al. demonstrated that the diffusion of ibuprofen increased for higher $\mathrm{pH}$ values, as a result of changing the electrostatic repulsion interaction and lowering the viscosity of mucus [15]. Interactions were related to the ionized state of the mucus and of ibuprofen. No change was observed for paracetamol, which is a unionized drug. Therefore, the effect on the charge interaction between the drug and mucus is more important than the effect of viscosity on diffusion in this study. Lieleg et al. found that electrostatic interactions were sensitive to the ion content of a solution. Given that the surface charges of synthetic particles or polymers were partially shielded by solubilized ions in buffer, the strength of the attractive or repulsive forces between diffusing particles and mucus depended on the salt content [66].

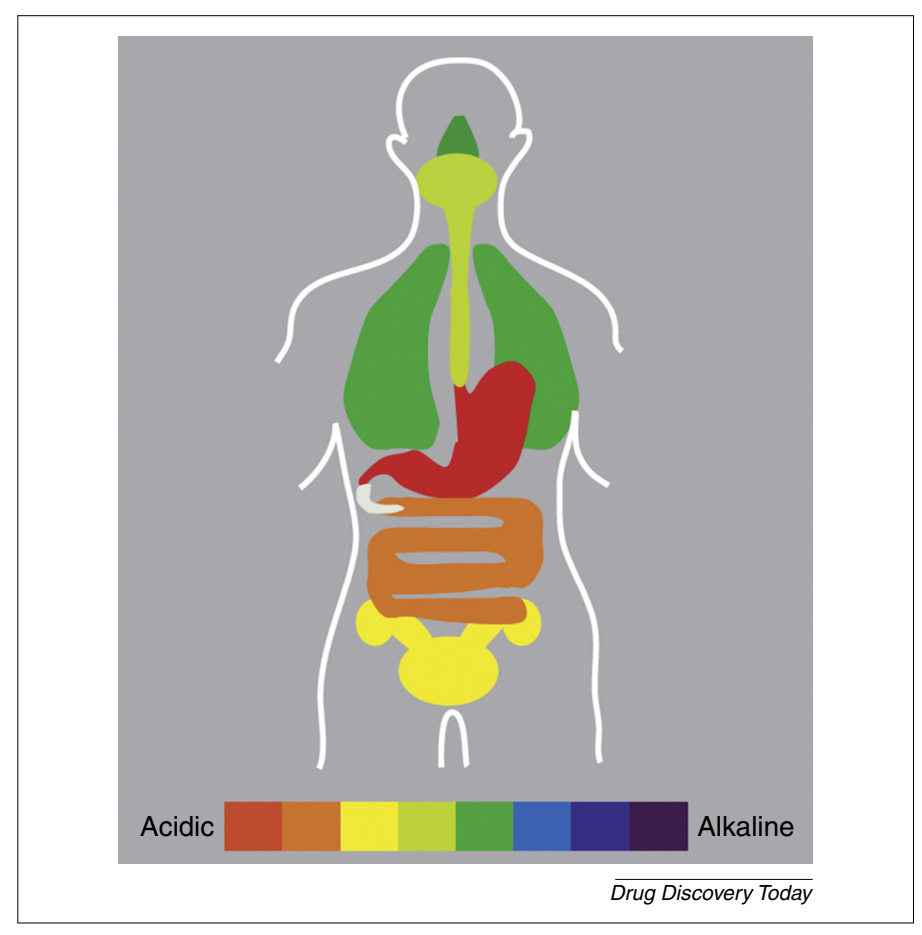

\section{FIGURE 3}

Mucus $\mathrm{pH}$ related to its localization in the body.

Figure adapted, with permission from Lieleg et al. [66].

The concentration of the studied particles is also important. For example, Lai et al. demonstrated that the addition of a high concentration of particles to CVM prevented their transport and caused the collapse of the mucus fibers, whereas a low concentration of particles did not cause bundling and allowed particle movements [19]. At high PS particle concentration (i.e. 10\%, v/ $\mathrm{w})$, hydrophobic interactions can cause the aggregation of mucin fibers in human cervical mucus (HCM) [26]. Similarly, Wang et al. observed that the effect of mucoadhesive nanoparticles on mucus depended on the particle concentration [76].

Therefore, drug and particle diffusion are sensitive to $\mathrm{pH}$, ionic force, viscosity, particle concentration, and the experimental conditions of diffusion studies (i.e. when and how long diffusion was observed). Given that experimental conditions can change between researchers and teams, comparisons between studies must therefore be made with caution.

\section{The specific case of colloid diffusion through mucus}

Knowing the relation between colloidal carrier properties and their ability to diffuse in mucus enables better design of these drug delivery systems. Here, we discuss each parameter that should be optimized to gain better colloid diffusion in mucus.

\section{Colloid design: size choice}

Most studies conclude that smaller particles move faster in mucus [52]. For example, Sanders et al. observed a difference in the transport of PS nanospheres through CFS, depending on particle size. Increasing particle size from $124 \mathrm{~nm}$ to $270 \mathrm{~nm}$ or $560 \mathrm{~nm}$ decreased the mean percent of nanospheres transported after $150 \mathrm{~min}$, caused mainly by stronger steric obstruction [29]. Similarly, Norris and Sinko observed the limited ability of particles $>0.5 \mu \mathrm{m}$ to diffuse through mucus [7]. By contrast, for Hosseinzadeh et al., a larger 


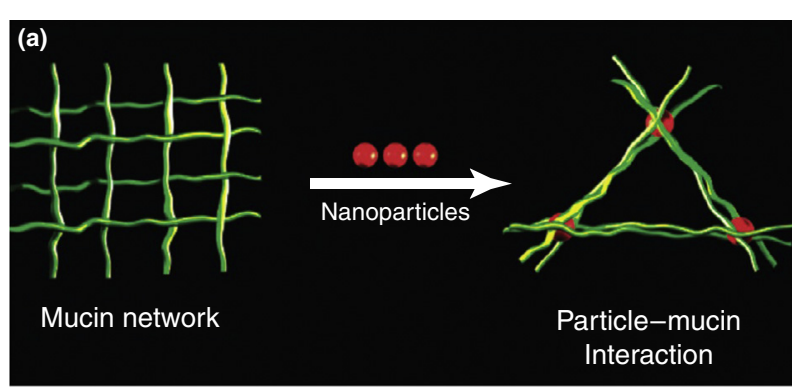

(b) Native (Untreated)

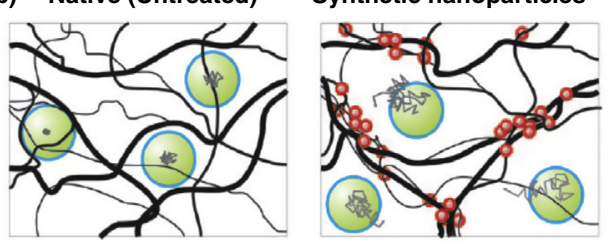

Probe nanoparticles (Non-mucoadhesive)

- Synthetic nanoparticles (Mucoadhesive)

- Sample trace of probes

- Mucus mesh elements

$\overline{\text { Drug Discovery Today }}$

FIGURE 4

(a) A mucus disruption induced by particles. A mucus fiber network is depicted on the left with the introduction of particles leading to their entanglement with mucus resulting in a change of the fiber network. (Figure reproduced, with permission, from McGill and Smyth [9].) (b) The potential effects of mucoadhesive particles on the mucus structure. Mucoadhesive particles can increase mucus pore sizes by bundling mucin fibers with adhesive interactions. (Figure reproduced, with permission, from Wang et al. [76].)

surface area was provided by smaller nanoparticle sizes, and increased adsorption in mucin, which led to higher mucoadhesive properties for mucoadhesive nanoparticles [70]. Other studies $[9,51]$ confirmed the relation between size and mucoadhesion, which influences particle diffusion.

Size effect might be the result of a steric obstruction of mucin mesh spacing that is linked to mucin interfiber spacing size. This size depends on the mucus model and the method used for its determination. For example, Saltzman et al. determined the geometric characteristic of HCM gels by scanning electron microscopy (SEM) and diffusion studies [21]. The probable interfiber spacing, assuming a random fiber arrangement, was $170 \mathrm{~nm}$ and the inferred interfiber spacing obtained by measuring diffusion coefficient was $150 \mathrm{~nm}$. Olmsted et al. predicted a mesh spacing of $100 \mathrm{~nm}$, by applying Amsdem's obstruction-scaling model to HCM and by electron microscopy [26]. Yudin et al. revealed that mucus has a fibrous structure with a 500-nm interfiber spacing between the primary elements and an additional finer structure with a spacing of approximately $100 \mathrm{~nm}$ [93]. Similarly, Kirch et al. observed by cryogenic SEM that horse respiratory mucus had large pores heterogeneously combined with very small pores [17], which is in accordance with findings for CVM [18]. In SEM images, normal HAM pores ranged from tens to hundreds of nanometers in diameter, with many pores $<100 \mathrm{~nm}$ [27].
Colloid design: considerations of surface properties

As we discuss below, surface properties such as electric charge, chemical moieties, hydrophilicity and/or lipophilicity have been showed to have a significant role in the ability of colloidal carriers for mucus diffusion. Thus, a negative relation between the diffusion of peptides and their lipophilic properties (i.e. $\log P$ ) in PIM has been observed [5]. Mistry et al. also determined that polysorbate-coated PS nanoparticles increased transport in mucus by increasing hydrophilicity [55]. A surface modification of the PS particle with pegylated polysorbate, increased transport through mucus not only by increasing the hydrophilicity, but also by reducing the negative charge of the PS particle. Wang et al. observed hydrophobic interactions between hydrophobic domains on the particles and the mucin fibers [20]. These interactions led to an attraction between particles and mucin. Similarly, Norris and Sinko observed that the amidine PS microspheres, which have the lowest hydrophobicity, also had the highest permeability through gastrointestinal mucin solution [7]. Thus, to avoid lipophilic interactions, the particle surface must be hydrophilic, although other properties have been shown to have a role in diffusion and different types of interaction are balance each other out. In the same study, the zeta potential was also shown to be a valuable indicator of the diffusion ability of PS particles, with a lower zeta potential favoring a higher diffusion ability. Repulsive electrostatic interactions were also observed between negatively charged particles and negatively charged mucin. However, if a particle was too attracted by mucin because of lipophilic or electrostatic interactions, the particle became entangled in mucus. By contrast, if a particle was too repulsed by electrostatic interaction, it was unable to diffuse through mucus. In the case of hydrophilic particles, diffusion is easier for particles that have no charge (i.e. neutral particles).

Dawson et al. prepared cationic particles by adding a cationic surfactant (PLGA-DDAB/DNA) to COOH PS-particles to enhance their hydrophilicity. They observed that cationic particles aggregated with mucus, which might have led to larger mucus pores and promoted more rapid transport for a fraction of particles [12]. Mura et al. modified PLGA nanoparticle surface charge with chitosan (CS), pluronic F68 (PF68), and poly(vinyl alcohol) (PVA) [24]. A positive zeta potential was obtained with PLGA/CS nanoparticles, whereas it remained almost neutral for PLGA/PVA nanoparticles, and was negative for PLGA/PF68 nanoparticles. PLGA nanoparticles exhibited a hydrophobic surface, which interacted with the hydrophobic domains of the mucin chains. PLGA/CS and PLGA/PVA nanoparticles became entrapped by mucus, whereas PLGA/PF68 nanoparticles diffused unimpeded between the mucin networks. Hydrophobic interactions were balanced by electrostatic repulsions. The coating with Pluronic ${ }^{\circledR}$ F127 (PF127) on PLGA particles led to a near-neutral surface charge, whereas coated particles diffused more freely in CRSM compared with uncoated particles [30]. The modification of liposomes with PF127 improved diffusion through native rat intestinal mucus, because of the distribution of the hydrophilic polyoxyethylene part of PF127 on the liposome surface [14]. As a consequence, hydrophobic and electrostatic interactions of the liposome with mucin were reduced.

Crater and Carrier showed significant differences between anionic and cationic particle mucus-penetrating capacities [8]. Particle 
mobility was inversely related to surface zeta potential. Sufficient surface coverage of anionic functionalities obtained with anionic (carboxylate or sulfate) particles suppressed the attractive interactions between the hydrophobic PS particle cores and mucin, whereas inadequate surface coverage with cationic (amine) particles resulted in increased hydrophobic interactions and led to the formation of particle aggregate. As a consequence, negative charge covering resulted in a significantly higher transport rate for particles exhibiting a hydrophobic core.

\section{Mucoadhesive molecules}

As discussed above, the literature has demonstrated evidence for a link between mucoadhesion and mucodiffusion. To some extent, mucoadhesive nano- or microparticles have more possibilities by which to diffuse, if they manage to avoid becoming entrapped in mucus because of interactions that are too strong. Thus, some studies have used mucoadhesive molecules associated with colloidal carriers to improve particle diffusion through mucus. Various novel mucoadhesive polymers have been developed, including lectins, thiolated polymers, bioadhesive nanopolymers, pluronics, alginate-polyethylene glycol acrylate and poloxomer [94]. For example, Ezpeleta et al. showed that lectin conjugates used for the delivery of hydrophobic drugs had an important affinity for mucin [73]. CS is another well-known mucoadhesive polysaccharide that forms disulfide bonds with cysteine-rich domains of mucus and also displays electrostatic interactions. Mucoadhesion effectiveness depends on polymer chemical features, such as MW, chain length, spatial arrangement, flexibility, hydration of polymer, hydrogen bonding, charge, and polymer concentration. Moghaddam et al. found better mucoadhesion with smaller nanoparticles with medium MW CS [95]. This polymer was modified by the addition of thiol group to obtain thiolated polymers called 'thiomers', capable of forming a thiolsulfide exchange reaction. Bravo-Osuna et al. developed a modified CS, called thiolated CS, which left particles still able to diffuse through the mucus [77]. Gradauer et al. also found that thiolated CS-coating doubled liposome mucoadhesion compared with uncoated liposomes [96]. The design of nanomicelles based on the acetylcysteine (NAC) functionalized CS-vitamin E succinate copolymer exhibited an ability to penetrate mucus [83]. As a consequence, NAC molecules increased the bioavailability of CS-vitamin E succinate nanomicelles, because of its good thiol activity. Petit et al. also found that CS enhanced nanoparticle mucoadhesion. Moreover, the mucoadhesion was enhanced twofold by the introduction of thiol groups on the surface of the CS nanoparticles [48]. A novel preactivated thiolated CS improved mucoadhesion compared with thiolated CS because of more active sulfhydryl moieties being available that protected thiol against early oxidation [71]. A novel amphiphilic copolymer was developed by Wang et al. by grafting glyceryl monostearate on CS [84]. This hydrophobic modification increased the mucoadhesion of the CS nanoparticles significantly $(P<0.05)$. Chen et al. compared the mucus penetration of liposomes modified with PF127 or CS [97]. They demonstrated that PF127-liposomes were inclined to penetrate the mucus and then to accumulate more effectively in intestinal tissue, owing to their more neutral and hydrophilic surface compared with CS liposomes and non-modified liposomes. Most CS liposomes were trapped in the mucus, resulting in limited mucus penetration.
Moreover, CS use is not without risk to the administration site, because CS has a tendency to form complexes with mucin and other proteins, which could cause major disturbances to the epithelium membrane [98].

\section{Coating particle with PEG}

To avoid hydrophobic and electrostatic interactions, mucus-penetrating particles (MPPs) were coated with PEG, a hydrophilic and uncharged polymer. This coating minimized efficiently particle adhesion to mucus constituents [30]. Griffiths et al. observed that non-ionic polymers, such as $10-\mathrm{kDa}$ MW or 100-kDA PEG did not interact with mucin, whereas dendrimers and polyethylenimine (PEI) exposed strong electrostatic (pH-dependent) interactions. Therefore, by designing polymer-based drug delivery systems, electrostatic interactions can be modulated to obtain good diffusion through mucus [10]. Similarly, the results of Tang et al. suggested that the sufficient PEG density of poly(sebacic acid) (PSA)-PEG particles provided rapid nanoparticle penetration of CVM and CFS [23]. PSA particles were strongly trapped by CVM, whereas PSA-PEG particles diffused unimpeded. Lai et al. showed that coating with 2-kDA PEG chains increased not only PS nanoparticle transport rates in CVM, but also the homogeneity of transport [19].

In agreement with findings in CVM [19], a dense covalent coating of low MW PEG led to particles penetrating more easily in other types of mucus, such as CFM [28], CRSM [30], and HAM [27]. Similarly, PEG-coated particles of 100, 200 and $500 \mathrm{~nm}$ penetrated mucus more rapidly than did uncoated particles of the same size [18]. Zabaleta et al. compared the apparent permeability through intestinal rat tissues of particles coated with different size of PEG: 2, 6 or $10 \mathrm{kDa} \mathrm{MW}$ [47]. The apparent permeability of particles pegylated with PEG of $2 \mathrm{kDa} \mathrm{MW}$ or $6 \mathrm{kDa}$ MW was 2.5 times higher than nanoparticles pegylated with PEG of $10 \mathrm{kDa}$ MW. A lower interaction between mucus layer and nanoparticles pegylated with PEG of lower MW explained the findings. Wang et al. also increased the coated particle displacements in CVM by a reduction in PEG from $10 \mathrm{kDa} \mathrm{MW}$ to $2 \mathrm{kDa}$ MW [20]. However, particles coated with $5 \mathrm{kDa}$ MW displayed rapid mucus-penetrating properties. These results indicated that a crucial MW threshold exists between 5 and $10 \mathrm{kDa}$. A small difference in the surface PEG coverage led to a 700-times decrease in the transport rate of PEG 2 kDA-PS particles with $40 \%$ PEG coverage, compared with the same particle covered with $65-70 \%$ PEG. Mert et al. observed that PLGA-vitamin E-PEG 1 kDa nanoparticles were as strongly trapped in CVM as uncoated PS nanoparticles, despite the coating, whereas PLGA/vitamin E-PEG $5 \mathrm{kDa}$ nanoparticles rapidly penetrated CVM. This was the result of inadequate surface coverage of 1-kDA PEG [25]. Similarly, mucoadhesion of DNA particles was not reduced by low MW PEG coatings, probably because of inadequate PEG surface coverage [85]. Thus, inadequate PEG surface density appears to be a crucial limiting factor for the development of MPP. These results completed the design requirement of PEG-coated MPP. In conclusion, suitable particles must exhibit: (i) PEG of sufficiently low MW and (ii) a sufficiently high density of PEG surface coverage.

The hydrophobic core of PS particles formed polyvalent adhesive interactions with hydrophobic domains along mucin fibers and possibly with other mucus constituents. Coating particles 
with PEG might reduce these particle-mucus adhesive interactions if the MW of PEG is too low to support adhesion by polymer interpenetration and hydrogen bonding [99-101]. PEG with low MW adopted a brush conformation that could facilitate the diffusion of particles in mucus by hindering the hydrophobic interactions $[99,100]$, as, for example, is the case for PEG of $2 \mathrm{kDa} M W$ or $6 \mathrm{kDa}$ MW. By contrast, the disposition of longer PEG chains (i.e. $10 \mathrm{kDa}$ ) was different at the nanoparticle surface and favored the interpenetration and interaction with the mucus fibers [101]. PEGs with a too low MW, for example $1 \mathrm{kDa}$, were distributed inside or physically adsorbed on the nanoparticle surface. The pegylated nanoparticles obtained had a conserved high affinity for the mucus [99]. Regardless of these considerations, biodegradable MPPs have been developed and tested in vitro [102] and in vivo [57]. However, rapid penetration of CVM by these MPPs with PEG MW from $1 \mathrm{kDa}$ to $10 \mathrm{kDa}$ showed that a large range of PEG MW can allow the preparation of muco-inert nanoparticles. The determination of PEG MW range can be affected by various factors, such as the particle size, core material, type of mucus and surface PEG density. MPPs improved mucus diffusion, vaginal drug distribution and retention without causing inflammation, and PEG improved the mucus penetration of other carriers, such as solid lipid nanoparticles [103].

\section{Diffusion enhancer}

McGill and Smyth treated mucus with functionalized PS nanoand microparticles to disrupt the mucus before molecule diffusion [9]. The disruption was significant, increasing permeation of fluorescein and rhodamine through different mucus models. Similarly, Wang et al. from the team of J. Hanes used high concentrations of mucoadhesive particles (MAP) (Fig. 4), which enlarged mucus mesh pores to increase muco-inert PEG-coated particle diffusion by tenfold [76]. The mucoadhesive properties of amine-modified PS particles sized $200 \mathrm{~nm}$ resulted from the hydrophobic core and positive charges. Exposure to MAP can be a dangerous strategy because it can significantly increase the risk of infection or toxicity by enhancing penetration by pathogens or other foreign particles with muco-inert surfaces. Ensign showed that hypotonic formulations improved epithelial surface distribution and retention of MPP [56].

Another strategy to disturb the mucus layer is to use mucolytic molecules, such as DNase and $\mathrm{N}$-acetylcysteine, to enhance nanoparticle diffusion through CFS [104]. Müller $e$ t al. functionalized particles with papain, a highly mucolytic enzyme, to reduce mucin crosslinks [105]. As a consequence, the application of these particles on PIM decreased mucus viscosity and improved particle diffusion.

\section{Concluding remarks}

Diffusion is a complex phenomenon that is sensitive to mucus composition and experimental parameters. To predict in vivo reality, the mucus selected for in vitro studies must be similar in composition and structure to the in vivo targeted mucus and the experimental parameters must be controlled carefully.

Mucus is an efficient barrier for particle diffusion because of physicochemical (hydrophobic, electrostatic, and hydrogen) interactions and steric occlusion related to its structure. Three strategies to improve diffusion through mucus have been described in the literature: (i) disruption of the mucus barrier; (ii) adhesive particles; and (iii) MPP [69], the importance of which has increased over the past few years [106].

In this Foundation Review, we have shown that the results for diffusion studies are often linked to the model used; therefore, a standard experimental protocol is needed to enable cross comparison of the colloids in terms of their ability to diffuse across mucus. The set-up of predictive models is also mandatory to enable the design of effective colloidal carriers that will diffuse easily through mucus, thus improving the performance of these new drug delivery systems.

\section{Acknowledgements}

A-C.G. is a fellow from Ethypharm supported by Association Nationale de la Recherche et de la Technologie (ANRT).

\section{References}

1 Kim, B.Y. et al. (2010) Nanomedicine. N. Engl. J. Med. 363, 2434-2443

2 Bertrand, N. and Leroux, J.C. (2012) The journey of a drug-carrier in the body: an anatomo-physiological perspective. J. Control. Release 161, 152-163

3 Khanvilkar, K. et al. (2001) Drug transfer through mucus. Adv. Drug Deliv. Rev. 48 173-193

4 Hida, K. et al. (2011) Common gene therapy viral vectors do not efficiently penetrate sputum from cystic fibrosis patients. PLOS ONE 6, e19919

5 Larhed, A.W. et al. (1997) Diffusion of drugs in native and purified gastrointestinal mucus. J. Pharm. Sci. 86, 660-665

6 Bhat, P.G. et al. (1995) The limiting role of mucus in drug absorption: drug permeation through mucus solution. Int. J. Pharm. 126, 179-187

7 Norris, D.A. and Sinko, P.J. (1997) Effect of size, surface charge, and

hydrophobicity on the translocation of polystyrene microspheres through gastrointestinal mucin. J. Appl. Polym. Sci. 63, 1481-1492

8 Crater, J.S. and Carrier, R.L. (2010) Barrier properties of gastrointestinal mucus to nanoparticle transport. Macromol. Biosci. 10, 1473-1483

9 McGill, S.L. and Smyth, H.D. (2010) Disruption of the mucus barrier by topically applied exogenous particles. Mol. Pharm. 7, 2280-2288

10 Griffiths, P.C. et al. (2010) PGSE-NMR and SANS studies of the interaction of model polymer therapeutics with mucin. Biomacromolecules 11, 120-125

11 Grubel, P. and Cave, D.R. (1998) Factors affecting solubility and penetration of clarithromycin through gastric mucus. Aliment. Pharmacol. Ther. 12, 569-576

12 Dawson, M. et al. (2004) Transport of polymeric nanoparticle gene carriers in gastric mucus. Biotechnol. Prog. 20, 851-857

13 Larhed, A.W. et al. (1998) The influence of intestinal mucus components on the diffusion of drugs. Pharm. Res. 15, 66-71

$14 \mathrm{Li}, \mathrm{X}$. et al. (2011) Novel mucus-penetrating liposomes as a potential oral drug delivery system: preparation, in vitro characterization, and enhanced cellular uptake. Int. J. Nanomedicine 6, 3151-3162

15 Shaw, L.R. et al. (2005) The influence of excipients on the diffusion of ibuprofen and paracetamol in gastric mucus. Int. J. Pharm. 290, 145-154

16 Bhat, P.G. et al. (1996) Drug diffusion through cystic fibrotic mucus: steady-state permeation, rheologic properties, and glycoprotein morphology. J. Pharm. Sci. 85, 624-630

17 Kirch, J. et al. (2012) Optical tweezers reveal relationship between microstructure and nanoparticle penetration of pulmonary mucus. Proc. Natl. Acad. Sci. U. S. A. $109,18355-18360$

18 Lai, S.K. et al. (2010) Nanoparticles reveal that human cervicovaginal mucus is riddled with pores larger than viruses. Proc. Natl. Acad. Sci. U. S. A. 107, 598-603

19 Lai, S.K. et al. (2007) Rapid transport of large polymeric nanoparticles in fresh undiluted human mucus. Proc. Natl. Acad. Sci. U. S. A. 104, 1482-1487

20 Wang, Y.Y. et al. (2008) Addressing the PEG mucoadhesivity paradox to engineer nanoparticles that "slip" through the human mucus barrier. Angew. Chem. Int. Ed. 47, 9726-9729 
21 Saltzman, W.M. et al. (1994) Antibody diffusion in human cervical mucus. Biophys. J. 66 (Pt 1), 508-515

22 Lai, S.K. et al. (2009) Altering mucus rheology to "solidify" human mucus at the nanoscale. PLOS ONE 4

23 Tang, B.C. et al. (2009) Biodegradable polymer nanoparticles that rapidly penetrate the human mucus barrier. Proc. Natl. Acad. Sci. U. S. A. 106, 19268-19273

24 Mura, S. et al. (2011) Biodegradable nanoparticles meet the bronchial airway barrier: how surface properties affect their interaction with mucus and epithelial cells. Biomacromolecules 12, 4136-4143

25 Mert, O. et al. (2012) A poly(ethylene glycol)-based surfactant for formulation of drug-loaded mucus penetrating particles. J. Control. Release 157, 455-460

26 Olmsted, S.S. et al. (2001) Diffusion of macromolecules and virus-like particles in human cervical mucus. Biophys. J. 81, 1930-1937

27 Schuster, B.S. et al. (2013) Nanoparticle diffusion in respiratory mucus from humans without lung disease. Biomaterials 34, 3439-3446

28 Suk, J.S. et al. (2009) The penetration of fresh undiluted sputum expectorated by cystic fibrosis patients by non-adhesive polymer nanoparticles. Biomaterials 30, 2591-2597

29 Sanders, N.N. et al. (2000) Cystic fibrosis sputum: a barrier to the transport of nanospheres. Am. J. Respir. Crit. Care Med. 162, 1905-1911

30 Lai, S.K. et al. (2011) Drug carrier nanoparticles that penetrate human chronic rhinosinusitis mucus. Biomaterials 32, 6285-6290

31 Lesuffleur, T. et al. (1991) Dihydrofolate reductase gene amplification-associated shift of differentiation in methotrexate-adapted HT-29 cells. J. Cell Biol. 115, 1409_ 1418

32 Behrens, I. et al. (2002) Comparative uptake studies of bioadhesive and nonbioadhesive nanoparticles in human intestinal cell lines and rats: the effect of mucus on particle adsorption and transport. Pharm. Res. 19, 1185-1193

33 Pontier, C. et al. (2001) HT29-MTX and Caco-2/TC7 monolayers as predictive models for human intestinal absorption: role of the mucus layer. J. Pharm. Sci. 90, 1608-1619

34 Lesuffleur, T. et al. (1993) Differential expression of the human mucin genes MUC1 to MUC5 in relation to growth and differentiation of different mucus-secreting HT-29 cell subpopulations. J. Cell Sci. 106, 771-783

35 Poquet, L. et al. (2008) Transport and metabolism of ferulic acid through the colonic epithelium. Drug Metab. Dispos. 36, 190-197

36 Behrens, I. et al. (2001) Transport of lipophilic drug molecules in a new mucussecreting cell culture model based on HT29-MTX cells. Pharm. Res. 18, 1138-1145

37 Mahler, G.J. et al. (2009) Characterization of Caco-2 and HT29-MTX cocultures in an in vitro digestion/cell culture model used to predict iron bioavailability. J. Nutr. Biochem. 20, 494-502

38 Meaney, C. and O'Driscoll, C. (1999) Mucus as a barrier to the permeability of hydrophilic and lipophilic compounds in the absence and presence of sodium taurocholate micellar systems using cell culture models. Eur. J. Pharm. Sci. 8, 167175

39 Schipper, N.G.M. et al. (1999) Chitosans as absorption enhancers of poorly absorbable drugs. 3. Influence of mucus on absorption enhancement. Eur. J. Pharm. Sci. 8, 335-343

40 Karlsson, J. et al. (1993) The mucus layer as a barrier to drug absorption in monolayers of human intestinal epithelial HT29-H goblet cells. Int. J. Pharm. 99, 209-218

41 Vllasaliu, D. et al. (2011) Barrier characteristics of epithelial cultures modelling the airway and intestinal mucosa: a comparison. Biochem. Biophys. Res. Commun. 415 $579-585$

42 Chen, X.M. et al. (2010) Defining conditions for the co-culture of Caco-2 and HT29-MTX cells using Taguchi design. J. Pharmacol. Toxicol. Methods 61, 334-342

43 Walter, E. et al. (1996) HT29-MTX/Caco-2 cocultures as an in vitro model for the intestinal epithelium: in vitro-in vivo correlation with permeability data from rats and humans. J. Pharm. Sci. 85, 1070-1076

44 Wikman-Larhed, A. and Artursson, P. (1995) Co-cultures of human intestinal goblet (HT29-H) and absorptive (Caco-2) cells for studies of drug and peptide absorption. Eur. J. Pharm. Sci. 3, 171-183

45 Wadell, C. et al. (1999) Nasal drug delivery - evaluation of an in vitro model using porcine nasal mucosa. Eur. J. Pharm. Sci. 7, 197-206

46 Takatsuka, S. et al. (2006) Enhancement of intestinal absorption of poorly absorbed hydrophilic compounds by simultaneous use of mucolytic agent and non-ionic surfactant. Eur. J. Pharm. Biopharm. 62, 52-58

47 Zabaleta, V. et al. (2012) Oral administration of paclitaxel with pegylated poly(anhydride) nanoparticles: permeability and pharmacokinetic study. Eur. J. Pharm. Biopharm. 81, 514-523

48 Petit, B. et al. (2012) The counterbalanced effect of size and surface properties of chitosan-coated poly(isobutylcyanoacrylate) nanoparticles on mucoadhesion due to pluronic F68 addition. Pharm. Res. 29, 943-952
49 Agüeros, M. et al. (2009) Combined hydroxypropyl- $\beta$-cyclodextrin and poly(anhydride) nanoparticles improve the oral permeability of paclitaxel. Eur. J. Pharm. Sci. 38, 405-413

50 Jin, Y. et al. (2011) Goblet cell-targeting nanoparticles for oral insulin delivery and the influence of mucus on insulin transport. Biomaterials 33, 1573-1582

$51 \mathrm{He}$, C. et al. (2012) Size-dependent absorption mechanism of polymeric nanoparticles for oral delivery of protein drugs. Biomaterials 33, 8569-8578

52 Szentkuti, L. (1997) Light microscopical observations on luminally administered dyes, dextrans, nanospheres and microspheres in the pre-epithelial mucus gel layer of the rat distal colon. J. Control. Release 46, 233-242

53 Lundin, P.D.P. et al. (1997) Enhancing effects of monohexanoin and two other medium-chain glyceride vehicles on intestinal absorption of desmopressin (dDAVP). J. Pharmacol. Exp. Ther. 282, 585-590

54 Xiao, W. et al. (2012) Biodistribution and pharmacokinetics of a telodendrimer micellar paclitaxel nanoformulation in a mouse xenograft model of ovarian cancer. Int. J. Nanomedicine 7, 1587-1597

55 Mistry, A. et al. (2009) Effect of physicochemical properties on intranasal nanoparticle transit into murine olfactory epithelium. J. Drug Target. 17, 543-552

56 Ensign, L.M. et al. (2013) Enhanced vaginal drug delivery through the use of hypotonic formulations that induce fluid uptake. Biomaterials 34, 6922-6929

57 Ensign, L.M. et al. (2012) Mucus-penetrating nanoparticles for vaginal drug delivery protect against herpes simplex virus. Sci. Transl. Med. 4, 138-179

58 Bhat, P.G. et al. (1996) Drug binding to gastric mucus glycoproteins. Int. J. Pharm. $134,15-25$

59 Boskey, E.R. et al. (2003) A self-sampling method to obtain large volumes of undiluted cervicovaginal secretions. Sex. Transm. Dis. 30, 107-109

60 Kararli, T.T. (1995) Comparison of the gastrointestinal anatomy, physiology, and biochemistry of humans and commonly used laboratory animals. Biopharm. Drug Dispos. 16, 351-380

61 Groo, A-C. et al. (2013) Fate of paclitaxel lipid nanocapsules in intestinal mucus in view of their oral delivery. Int. J. Nanomedicine 8, 4291-4302

62 Fogg, F.J. et al. (1996) Characterization of pig colonic mucins. Biochem. J. 316, $937-$ 942

63 Verdugo, P. et al. (1987) Molecular mechanism of product storage and release in mucin secretion. II. The role of extracellular $\mathrm{Ca}^{++}$. Biorheology 24, 625-633

64 Chen, E.Y. et al. (2010) A new role for bicarbonate in mucus formation. Am. J. Physiol. Lung Cell. Mol. Physiol. 299, L542-L549

65 Aitken, M.L. and Verdugo, P. (1989) Donnan mechanism of mucin release and conditioning in goblet cells: the role of polyions. Symp. Soc. Exp. Biol. 43, 73-80

66 Lieleg, O. et al. (2010) Characterization of particle translocation through mucin hydrogels. Biophys. J. 98, 1782-1789

67 Matsui, H. et al. (2006) A physical linkage between cystic fibrosis airway surface dehydration and Pseudomonas aeruginosa biofilms. Proc. Natl. Acad. Sci. U. S. A. 103 , 18131-18136

68 Ensign, L.M. et al. (2013) Ex vivo characterization of particle transport in mucus secretions coating freshly excised mucosal tissues. Mol. Pharm. 10, 2176-2182

69 Ensign, L.M. et al. (2012) Oral drug delivery with polymeric nanoparticles: the gastrointestinal mucus barriers. Adv. Drug Deliv. Rev. 64, 557-570

70 Hosseinzadeh, H. et al. (2012) Chitosan-Pluronic nanoparticles as oral delivery of anticancer gemcitabine: preparation and in vitro study. Int. J. Nanomedicine 7, $1851-1863$

71 Mûller, C. et al. (2013) Thiopyrazole preactivated chitosan: combining mucoadhesion and drug delivery. Acta Biomater. 9, 6585-6593

72 Jain, S. et al. (2012) Polyelectrolyte stabilized multilayered liposomes for oral delivery of paclitaxel. Biomaterials 33, 6758-6768

73 Ezpeleta, I. et al. (1999) Preparation of Ulex europaeus lectin-gliadin nanoparticle conjugates and their interaction with gastrointestinal mucus. Int. J. Pharm. 191, $25-32$

74 Apgar, J. et al. (2000) Multiple-particle tracking measurements of heterogeneities in solutions of actin filaments and actin bundles. Biophys. J. 79, 1095-1106

75 Suh, J. et al. (2005) Real-time multiple-particle tracking: applications to drug and gene delivery. Adv. Drug Deliv. Rev. 57, 63-78

76 Wang, Y.Y. et al. (2011) Mucoadhesive nanoparticles may disrupt the protective human mucus barrier by altering its microstructure. PLOS ONE 6, e21547

77 Bravo-Osuna, I. et al. (2008) Specific permeability modulation of intestinal paracellular pathway by chitosan-poly(isobutylcyanoacrylate) core-shell nanoparticles. Eur. J. Pharm. Biopharm. 69, 436-444

78 Kowapradit, J. et al. (2012) Methylated N-(4-N,N-dimethylaminobenzyl) chitosan coated liposomes for oral protein drug delivery. Eur. J. Pharm. Sci. 47, 359-366

79 Chakravarthi, S.S. and Robinson, D.H. (2011) Enhanced cellular association of paclitaxel delivered in chitosan-PLGA particles. Int. J. Pharm. 409, 111-120

80 Occhipinti, P. and Griffiths, P.C. (2008) Quantifying diffusion in mucosal systems by pulsed-gradient spin-echo NMR. Adv. Drug Deliv. Rev. 60, 1570-1582 
81 Groo, A.-C. et al. (2014) Development of 2D and 3D mucus models and their interactions with mucus penetrating paclitaxel loaded lipid nanocapsules. Pharm. Res. http://dx.doi.org/10.1007/s11095-013-1280-4 (in press)

82 Prego, C. et al. (2006) Efficacy and mechanism of action of chitosan nanocapsules for oral peptide delivery. Pharm. Res. 23, 549-556

83 Lian, H. et al. (2013) Enhanced oral delivery of paclitaxel using acetylcysteine functionalized chitosan-vitamin E succinate nanomicelles based on a mucus bioadhesion and penetration mechanism. Mol. Pharm. 10, 3447-3458

84 Wang, L. et al. (2013) Exploration of hydrophobic modification degree of chitosanbased nanocomplexes on the oral delivery of enoxaparin. Eur. J. Pharm. Sci. 50, 263-271

85 Boylan, N.J. et al. (2012) Highly compacted DNA nanoparticles with low MW PEG coatings: in vitro, ex vivo and in vivo evaluation. J. Control. Release 157, 72-79

86 Sanders, N.N. et al. (2000) The physical properties of biogels and their permeability for macromolecular drugs and colloidal drug carriers. J. Pharm. Sci. 89, 835-849

87 Gordon, A.R. (1945) The diaphragm cell method of measuring diffusion. Ann. N. Y Acad. Sci. 46, 285-308

88 Dainty, J. and House, C.R. (1966) An examination of the evidence for membrane pores in frog skin. J. Physiol. 185, 172-184

89 Dainty, J. and House, C.R. (1966) Unstirred layers in frog skin. J. Physiol. 182, 66-78

90 Korjamo, T. et al. (2009) Analysis of unstirred water layer in in vitro permeability experiments. J. Pharm. Sci. 98, 4469-4479

91 Dainty, J. (1963) Water Relations of Plant Cells. London Academic Press

92 Cao, X. et al. (1999) pH-dependent conformational change of gastric mucin leads to sol-gel transition. Biophys. J. 76, 1250-1258

93 Yudin, A.I. et al. (1989) Human cervical mucus and its interaction with sperm: a fine-structural view. Biol. Reprod. 40, 661-671

94 Mythri, G. et al. (2011) Novel mucoadhesive polymers - a review. JAPS 1, 37-42
95 Moghaddam, F.A. et al. (2009) Preparation and in vitro evaluation of mucoadhesion and permeation enhancement of thiolated chitosan-pHEMA coreshell nanoparticles. Nanomedicine 5, 208-215

96 Gradauer, K. et al. (2012) Chemical coupling of thiolated chitosan to preformed liposomes improves mucoadhesive properties. Int. J. Nanomedicine 7, 2523-2534

97 Chen, D. et al. (2013) Comparative study of Pluronic $\left({ }^{\circledR}\right)$ F127-modified liposomes and chitosan-modified liposomes for mucus penetration and oral absorption of cyclosporine A in rats. Int. J. Pharm. 449, 1-9

98 Silva, C.A. et al. (2012) Interaction of chitosan and mucin in a biomembrane model environment. J. Colloid Interface Sci. 376, 289-295

99 Yoncheva, K. et al. (2005) Pegylated nanoparticles based on poly(methyl vinyl ether-co-maleic anhydride): preparation and evaluation of their bioadhesive properties. Eur. J. Pharm. Sci. 24, 411-419

100 Yoncheva, K. et al. (2007) Evaluation of bioadhesive potential and intestinal transport of pegylated poly(anhydride) nanoparticles. Int. J. Pharm. 334, 156-165

101 Huang, Y. et al. (2000) Molecular aspects of muco- and bioadhesion: tethered structures and site-specific surfaces. J. Control. Release 65, 63-71

$102 \mathrm{Xu}, \mathrm{Q}$. et al. (2013) Scalable method to produce biodegradable nanoparticles that rapidly penetrate human mucus. J. Control. Release 170, 279-286

103 Yuan, H. et al. (2013) Improved transport and absorption through gastrointestinal tract by PEGylated solid lipid nanoparticles. Mol. Pharm. 10, 1865-1873

104 Broughton-Head, V.J. et al. (2007) Actin limits enhancement of nanoparticle diffusion through cystic fibrosis sputum by mucolytics. Pulm. Pharmacol. Ther. 20, 708-717

105 Müller, C. et al. (2013) Preparation and characterization of mucus-penetrating papainpoly(acrylic acid) nanoparticles for oral drug delivery applications. $J$. Nanopart. Res. 15, 1-13

106 Ensign, L.M. et al. (2012) Mucus penetrating nanoparticles: biophysical tool and method of drug and gene delivery. Adv. Mater. 24, 3887-3894 